ORIGINAL

\title{
Utilización de la ventilación mecánica no invasiva en neumonía grave por virus $\mathrm{H} 1 \mathrm{~N} 1$
}

\author{
A. Belenguer-Muncharaz*, R. Reig-Valero, S. Altaba-Tena, P. Casero-Roig \\ y A. Ferrándiz-Sellés
}

Servicio de Medicina Intensiva, Hospital General Castellón, Castellón, España

Recibido el 23 de enero de 2011; aceptado el 4 de abril de 2011

Disponible en Internet el 19 de mayo de 2011

\author{
PALABRAS CLAVE \\ Gripe A (H1N1); \\ Neumonía; \\ Ventilación no \\ invasiva
}

\begin{abstract}
Resumen
Objetivo: Análisis del empleo de la VMNI en nuestra serie de pacientes ingresados en la unidad de cuidados intensivos ( $\mathrm{UCl}$ ) afectados por nuevo virus de la gripe $\mathrm{A}$ (H1N1), en especial aquellos afectados por neumonía con insuficiencia respiratoria aguda (IRA) hipoxémica grave, observando la necesidad de intubación, mejoría clínico-gasométrica, desarrollo de complicaciones, mortalidad, estancia en UCI y hospitalaria.

Diseño: Estudio retrospectivo observacional.

Ámbito: UCl del Hospital General de Castellón.

Pacientes: Pacientes ingresados en la unidad con neumonía primaria o secundaria, con IRA de predominio hipoxémico.

Intervenciones: Se empleó CPAP de Boussignac, sistema Helmet y BiPAP Vision.

Resultados: De un total de 10 pacientes ingresados con infección por gripe A H1N1, se empleó la VMNI en 7 (70\%) pacientes con un fracaso del 28\% (una agudización de asma y otra insuficiencia ventilatoria con obstrucción de vía aérea). Dentro del grupo hipoxémico analizado (5 pacientes), la efectividad de la VMNI fue del $100 \%$ en cuanto a mejoría gasométrica y clínica, evitando la intubación de todos estos pacientes. Asimismo, no se produjo ninguna muerte tanto en $\mathrm{UCI}$ como en el hospital. La duración (mediana) de la ventilación fue de 6 (4-11) días y la estancia en $\mathrm{UCI}$, de 9 (7-11) días. La tasa de complicaciones fue pequeña (una infección de orina). La tolerancia de la VMNI fue aceptable, destacando el ruido producido por la CPAP. No se produjo ningún contagio en el personal sanitario.

Conclusiones: A la luz de los resultados, se podría plantear un mayor empleo de la VMNI ante futuras epidemias.

(C) 2011 Elsevier España, S.L. y SEMICYUC. Todos los derechos reservados.
\end{abstract}

\footnotetext{
* Autor para correspondencia.

Correo electrónico: belenguer_alb@gva.es (A. Belenguer-Muncharaz).
} 


\section{KEYWORDS}

Influenza A virus

(H1N1);

Pneumonia;

Noninvasive

ventilation

\section{Noninvasive mechanical ventilation in severe pneumonia due to H1N1 virus}

\begin{abstract}
Objective: The use of noninvasive mechanical ventilation was evaluated in our series of patients admitted to our ICU with pneumonia due to influenza A virus $\mathrm{H} 1 \mathrm{~N} 1$, assessing the need for intubation, arterial blood gases and clinical improvement, the development of complications and ICU and hospital stay.

Design: Retrospective and observational study.

Setting: ICU of Castellón University General Hospital (Castellón, Spain).

Population: Patients admitted to ICU with pneumonia due to influenza A virus H1N1 and acute hypoxemic respiratory failure.

Interventions: Boussignac CPAP, Helmet system and BiPAP Vision ${ }^{\circledR}$ were used.

Results: Five of 10 patients with pneumonia and hypoxemia were analyzed, showing $100 \%$ effectiveness of noninvasive mechanical ventilation in terms of clinical and arterial blood gas improvement, and avoiding intubation in all cases. There were no patient deaths in ICU or in hospital. The duration (median) of ventilation was 6 (4-11) days, with an ICU stay of 9 (7-11) days. The number of complications was low (except for urinary tract infection due to Pseudomonas aeruginosa), and only the noise produced by CPAP was underscored. There were no infections among the staff.

Conclusions: Based on our results, increased use of noninvasive mechanical ventilation in future epidemics coujld be proposed.

(c) 2011 Elsevier España, S.L. and SEMICYUC. All rights reserved.
\end{abstract}

\section{Introducción}

La reciente pandemia producida por el nuevo virus de la gripe A (H1N1) ha supuesto una prueba importante para los sistemas sanitarios. Pese a la alarma inicial, la realidad ha demostrado que la infección ha afectado de un modo menor a lo estimado inicialmente. Pese a ello, los pacientes más graves, con importante afección orgánica, han precisado soporte en las unidades de cuidados intensivos $(\mathrm{UCl})$. Los estudios publicados ${ }^{1-3}$ han mostrado que los pacientes ingresados en $\mathrm{UCl}$ con infección probable o confirmada desarrollaron disfunción multiorgánica, destacando la insuficiencia respiratoria aguda (IRA) hipoxémica grave como alteración fisiopatológica que obligó a proporcionar soporte ventilatorio en un porcentaje de la población afecta que osciló entre el 71,8 y el $93 \%^{1-3}$, siendo la ventilación invasiva (VMI) la modalidad más empleada como primera opción terapéutica $(76,2 \%)$. Entre los que recibieron ventilación mecánica no invasiva (VMNI), el fracaso se situó entre el 75 y el $85 \% 1,2$.

La VMNI presenta menor tasa de complicaciones que la VMI (sobre todo por la menor incidencia de neumonía asociada a la ventilación mecánica), a la vez que permite una mayor tolerancia del paciente, ya que le permite hablar, comer, eliminar secreciones, etc. La utilización de la VMNI en pacientes con IRA hipoxémicos ha mostrado buenos resultados, por cuanto que mejora la oxigenación, reduce la fatiga, evita la intubación en un alto porcentaje de pacientes y reduce la mortalidad ${ }^{4-6}$. Pese a los beneficios mostrados, no se ha establecido una indicación para su empleo en la IRA hipoxémica ${ }^{7,8}$.

En el presente estudio, analizamos el empleo de la VMNI en pacientes con neumonía e hipoxemia grave de nuestra serie de pacientes afectados por el nuevo virus de la gripe A (H1N1) y que requirieron del ingreso en UCl. Para ello, analizamos la incidencia de la VMNI, el fracaso y mortalidad del grupo no invasivo, así como la evolución desde el punto de vista tanto clínico (medido por frecuencia cardíaca y respiratoria) como gasométrico (mediante el cociente $\mathrm{pO}_{2} / \mathrm{FiO}_{2}$ ).

\section{Pacientes y métodos}

Tipo de estudio: observacional retrospectivo.

\section{Pacientes}

Se incluyó a los pacientes ingresados en nuestra unidad desde agosto de 2009 y hasta enero de 2010 con el diagnóstico de presunción, sospecha o confirmación de infección por el nuevo virus de la gripe A (H1N1), y que desarrollaron neumonía primaria o secundaria (a neumonía bacteriana previa) con IRA hipoxémica.

Se definió neumonía comunitaria como infección del tracto respiratorio inferior con presencia de opacidades en la radiografía de tórax, signos y síntomas de infección respiratoria (fiebre, tos, dolor pleurítico, leucocitosis o leucopenia y presencia o ausencia de secreciones) ${ }^{9}$. Se definió neumonía primaria en los casos que se demostró infección por el nuevo virus de la gripe A (H1N1) y sin aislamiento de Legionella spp. o Streptococcus pneumoniae en sangre o muestras pulmonares, o sus antígenos en orina. En caso contrario, se consideraron neumonías secundarias ${ }^{10}$. Las neumonías fueron consideradas graves si cumplían los criterios establecidos por la ATS/IDSA ${ }^{9}$. Se estableció la definición clásica de insuficiencia respiratoria aguda (IRA) de inicio como la presencia de frecuencia respiratoria superior a 30 respiraciones/min, presión parcial de oxígeno $\left(\mathrm{PaO}_{2}\right)<60 \mathrm{mmHg}$ o presión parcial de anhídrido carbónico 
$\left(\mathrm{PaCO}_{2}\right)>45 \mathrm{mmHg}$. Dentro del grupo de pacientes con IRA hipoxémica $\left(\mathrm{PaO}_{2}<60 \mathrm{mmHg}\right.$ ), dichos pacientes cumplían los criterios de lesión pulmonar aguda (LPA) o síndrome de distrés respiratorio del adulto (SDRA).

Por lo tanto, se analizó a los pacientes con IRA hipoxémica con neumonía primaria o secundaria que, sin contraindicaciones para la aplicación de la $\mathrm{VMNI}^{7}$, se encontraban en condiciones para recibir dicha asistencia ventilatoria no invasiva.

Fueron excluidos del análisis final los pacientes que presentaban, a su ingreso, cuadro de IRA de predominio hipercápnico, además de no presentar imágenes radiológicas compatibles con infiltrados alveolares difusos e hipoxemia grave $\left(\mathrm{PaO}_{2}<60 \mathrm{mmHg}\right)$.

\section{Monitorización}

Al ingreso del paciente en la unidad, se canalizaba vía arterial y venosa central, para monitorización hemodinámica menos invasiva empleando el sistema PiCCO (PULSION Medical Systems, Munich, Alemania). La saturación transcutánea de oxígeno $\left(\mathrm{SatcO}_{2}\right)$ se monitorizó a través de pulsioxímetro Oxisensor Nellcor II D-25 (Nellcor ${ }^{\circledR}$ Puritan Bennet Inc., Decasanton, CA, Estados Unidos) conectado a monitor de cabecera Infinity Delta (Dräger Medical System, Danver, Estados Unidos). Las muestras sanguíneas arteriales para determinaciones gasométricas se procesaron en cooxímetro ABL560 (Radiometer Medical A/S ${ }^{\circledR}$, Copenhagen, Dinamarca). Se recogieron muestras de esputo, orina y hemocultivos para identificar causantes del proceso neumónico. Se procedió a frotis nasal y faríngeo, determinación de antigenemia y reacción en cadena de la polimerasa (PCR), como protocolo ante pacientes con sospecha de infección viral por $\mathrm{H} 1 \mathrm{~N} 1$.

A su ingreso y durante su estancia en la unidad, se recogieron datos de filiación, diagnóstico, estancia en $\mathrm{UCl}$ y hospitalaria, duración de ventilación mecánica, sistemas de estimación de gravedad a través del Acute Physiology And Chronic Health Evaluation (APACHE II) y Sequential Organ Failure Assessment (SOFA), así como de los correspondientes parámetros hemodinámicos, respiratorios y gasométricos (incluyendo en situación basal y a la $1 .^{\mathrm{a}}, 2 .^{\mathrm{a}}, 8 .^{\mathrm{a}}, 24 .^{\mathrm{a}}$ y $48 .^{\mathrm{a}}$ horas de iniciada la ventilación). Se recogieron las complicaciones que se produjeron a lo largo de la estancia en la unidad como: intubación orotraqueal, neumotórax, fracaso renal agudo (con o sin hemofiltración), situación de fracaso multiorgánico, infecciones nosocomiales y mortalidad. Los pacientes fueron interrogados sobre las complicaciones que pudiesen ser atribuidas a la VMNI (claustrofobia, aerofagia, insomnio, cefalea, ruido).

Como método de VMNI se emplearon los sistemas de presión continua en la vía aérea (CPAP), bien con sistema Helmet CaStar (StarMed; Mirandola, Módena, Italia) o bien mediante sistema de Boussignac-Vygon ${ }^{\circledR}$. El sistema Helmet se conectó a un sistema de CPAP Whisperflow ${ }^{\circledR}$ con un generador de alto flujo de oxígeno (hasta 150 l/min), y en el otro puerto del Helmet se colocó una pieza de presión espiratoria al final de la espiración (PEEP), a fin de conseguir una presión supraatmosférica durante todo el ciclo respiratorio.

En ambos sistemas de CPAP, los valores iniciales empleados fueron de $\mathrm{FiO}_{2}, 1$, y PEEP, $5-15 \mathrm{cmH}_{2} \mathrm{O}$ hasta conseguir mejoría clínica (disminución de frecuencia respiratoria, cardíaca) y/o $\mathrm{SatcO}_{2}$ del $94-96 \%$.

El dispositivo de VMNI empleado fue la BiPAP Vision (Respironics Inc. ${ }^{\circledR}$, Pennsylvania, Estados Unidos) conectado a mascarilla orofacial o facial completa o face Total face ${ }^{\circledR}$ (Respironics Inc. ${ }^{\circledR}$, Pennsylvania, Estados Unidos) y conectada a sistema de humidificación activa (Fischer and Payckel Healtcare ltd.).

Procedimiento: el paciente era ingresado en boxes individuales cerrados donde todo el personal asistencial adoptaba las medidas de protección frente al contagio establecidas por el Comité Hospitalario de Control de la Epidemia (guantes, bata, gorro y mascarillas N95).

Se explicaba al paciente el procedimiento que se le iba a realizar (VMNI o CPAP), procediendo a la colocación de cada dispositivo por parte de varios miembros del equipo. Se seleccionó la mascarilla según la situación clínica del paciente y tamaño acorde con la anatomía de la cara. Una vez iniciada la ventilación, se aumentó progresivamente el soporte ventilatorio con PEEP y presión soporte por encima de PEEP (PS), hasta conseguir unos volúmenes de $5-7 \mathrm{ml} / \mathrm{kg}$ y frecuencia respiratoria de $25-28 \mathrm{rpm}$ de tal manera que en la primera hora de soporte se alcanzase un mínimo de PS de $10-15 \mathrm{cmH}_{2} \mathrm{O}$ y una PEEP mínima de $5-8 \mathrm{cmH}_{2} \mathrm{O}$. La concentración de oxígeno se aumentó hasta conseguir una mejoría clínica (disminución de frecuencia respiratoria y frecuencia cardíaca) y/o una $\mathrm{SatcO}_{2}$ del 94-96\%. Una vez conseguida la colaboración del paciente y la suficiente adaptabilidad, se ajustó la mascarilla mediante tiras ajustables. Posteriormente, se evaluó el grado de tolerancia del paciente, tanto clínica como sicológica.

La indicación de intubación orotraqueal en pacientes sometidos a VMNI se sentaba ante la presencia de evidentes signos de trabajo respiratorio (taquipnea, uso de musculatura accesoria, disociación toracoabdominal, encefalopatía), acidosis metabólica láctica progresiva o disfunción multiorgánica. No se consideró exclusivamente la hipoxemia como criterio de intubación, puesto que estos enfermos presentaban una hipoxemia grave por su enfermedad de base.

Los pacientes ingresados en nuestra unidad fueron notificados como casos al registro nacional de gripe A promovido por el Grupo de Trabajo de Enfermedades Infecciosas (GTEI) de la SEMICYUC ${ }^{10}$.

\section{Consentimiento}

El protocolo del estudio fue aprobado por el Comité Asistencial de Ensayos Clínicos y se solicitó consentimiento escrito a los pacientes incluidos en el estudio.

\section{Analisis estadístico}

Se empleó el paquete estadístico SPSS 11.0 para el análisis estadístico. En la comparación de las variables cuantitativas se empleó el método de la $\mathrm{t}$ de Student o la $\mathrm{U}$ de MannWhitney, según muestras paramétricas o no paramétricas. Para las variables cualitativas se utilizó la prueba de la $\chi^{2}$ con el test exacto de Fisher (de dos colas) cuando el número de casos era inferior a 5. Significación estadística si $p<0,05$. 
Tabla 1 Características básicas de los pacientes a su ingreso en $\mathrm{UCI}$

\begin{tabular}{|c|c|c|}
\hline Variables & $\begin{array}{l}\text { Muestra } \\
\text { total } \\
(\mathrm{n}=10)\end{array}$ & $\begin{array}{l}\text { Muestra de } \\
\text { estudio } \\
(n=5)\end{array}$ \\
\hline Hombres $(n=10)$ & $5(50)$ & $3(60)$ \\
\hline Edad, años & $38[27-47]$ & $45[27-48]$ \\
\hline SOFA & $4[3-6]$ & $4[3-4]$ \\
\hline APACHE II & 8 [7-16] & $8[6-12]$ \\
\hline Fallo multiorgánico al ingreso & $1(10)$ & 0 \\
\hline \multicolumn{3}{|l|}{ Procedencia } \\
\hline Urgencias & $7(70)$ & $4(80)$ \\
\hline UCl otro hospital & $2(20)$ & \\
\hline Planta & $1(10)$ & $1(20)$ \\
\hline \multicolumn{3}{|l|}{ Comorbilidades } \\
\hline Embarazo & $2(20)$ & 0 \\
\hline Hipertensión arterial & $1(10)$ & $1(20)$ \\
\hline Tabaco & $1(10)$ & $1(20)$ \\
\hline Obesidad & $4(40)$ & $4(80)$ \\
\hline Enfermedad de Castleman & $1(10)$ & 0 \\
\hline \multicolumn{3}{|l|}{ Tipo de afección pulmonar } \\
\hline Neumonía viral primaria & $7(70)$ & $5(100)$ \\
\hline Agudización de asma & $1(10)$ & \\
\hline Insuficiencia ventilatoria & $1(10)$ & \\
\hline Intoxicación farmacológica & $1(10)$ & \\
\hline \multicolumn{3}{|l|}{ Terapia ventilatoria al ingreso } \\
\hline VMNI & $7(70) / 2(28)$ & $5(100)$ \\
\hline Neumonía viral primaria & $5(71) / 0$ & $5(100)$ \\
\hline Agudización de asma & $1(14,5) / 1$ & 0 \\
\hline Insuficiencia ventilatoria & $1(14,5) / 1$ & 0 \\
\hline VMI & $2(20)$ & 0 \\
\hline Sin ventilación & $1(10)$ & 0 \\
\hline Mortalidad & $1(10)$ & 0 \\
\hline
\end{tabular}

VMNI, ventilación mecánica no invasiva; VMI, ventilación mecánica invasiva.

Los datos expresan $\mathrm{n}(\%), \mathrm{n}$ total $(\%) / \mathrm{n}$ fracaso total $(\%) \mathrm{o}$ mediana [índice intercuartílico 25-75].

Se realizó análisis de muestras repetidas de variables cuantitativas para pruebas no paramétricas (test de Friedman) evaluando el efecto de la VMNI en las variables hemodinámicas y respiratorias.

\section{Resultados}

Se notificó al Registro Nacional de Gripe A, durante el periodo de agosto de 2009 a enero de 2010, un total de 10 pacientes, de los que 7 presentaban neumonía viral primaria, 2 presentaban insuficiencia ventilatoria por agudización de asma, uno en el contexto de enfermedad de Castleman, y el último, intoxicación medicamentosa con bajo nivel de conciencia inicial que obligó a su intubación en domicilio (tabla 1). La mayoría eran jóvenes, sin enfermedad de base, destacando el sobrepeso y el embarazo en 2 pacientes como antecedentes. Siete (70\%) pacientes procedían de urgencias, otros 2 pacientes procedían de otros hospitales y el último procedía de planta de hospitalización. Una vez ingresados, se instauró inicialmente soporte ventilatorio no invasivo en 7 (70\%) pacientes, mientras que 2 pacientes ya se encontraban intubados a su ingreso, y en otro caso, la óptima situación respiratoria de la paciente permitió no instaurar medida alguna de soporte ventilatorio.

Por lo que respecta al resto de los parámetros fisiológicos en situación basal (tabla 2), debemos destacar la importante hipoxemia de inicio, la importante afección de la radiografía de tórax y la presencia de alteraciones en la función hepática, con aumento de enzimas de citólisis. Todos los pacientes recibieron (o habían recibido), a su ingreso en la unidad, tratamiento antibiótico empírico con ceftriaxona, claritromicina y oseltamivir (en dosis de $150 \mathrm{mg} / 12 \mathrm{~h}$ ), excepto el paciente con broncoaspiración por intoxicación farmacológica en el que no había sospecha de infección por gripe A.

La mortalidad general fue de una paciente (10\%) que ingresó en nuestra unidad procedente de otro centro en situación de fallo multiorgánico, y falleció a las $24 \mathrm{~h}$. Dos pacientes $(30 \%)$ sometidos a VMNI fracasaron durante su estancia en la unidad, uno de ellos presentaba agudización grave del asma que evolucionó en pocas horas a insuficiencia ventilatoria grave con acidosis respiratoria por broncospasmo, y la otra paciente, con enfermedad de Castleman, presentó obstrucción de vía aérea superior por tejido inflamatorio supraglótico (por su enfermedad de base) que obligó a su intubación urgente a los 3 días de iniciar la VMNI. En ambos pacientes destacaba a su ingreso la acidosis respiratoria sobre la hipoxemia, junto con mínimos infiltrados pulmonares en la radiografía de tórax. Por estas razones se decidió excluirlos del estudio de los pacientes propiamente hipoxémicos.

La comparación de variables fisiológicas entre la muestra total y la muestra de los 5 pacientes con insuficiencia respiratoria hipoxémica no mostró diferencias significativas (tablas 1 y 2). La mediana de tiempo en la muestra total desde el inicio de los síntomas hasta su ingreso en el hospital fue de 3 (2-6) días y 4 (2-8) días, respectivamente, y para el grupo de estudio fue de 5 días en ambas variables de tiempo analizadas, sin diferencias en ambos grupos (tabla 3).

En el grupo hipoxémico, en 3 pacientes se utilizó CPAP con sistema Helmet ${ }^{\circledR}$, otro recibió VMNI y el último, una combinación de CPAP de Boussignac ${ }^{\circledR}$, pasando posteriormente a VMNI ante la ausencia de mejoría significativa. Es de destacar que ninguno de ellos requirió intubación orotraqueal (éxito del $100 \%$ ), lo que condicionó unos excelentes resultados en cuanto que ninguno de ellos falleció en $\mathrm{UCI}$ ni a su alta hospitalaria.

Desde el punto de vista clínico, se pudo apreciar mejora progresiva del intercambio gaseoso (fig. 1) y de la situación clínica del paciente medida por la frecuencia respiratoria (fig. 2) y por la frecuencia cardíaca (fig. 3), que mostró significación estadística $(\mathrm{p}<0,05)$.

La duración del soporte ventilatorio y de las estancias en $\mathrm{UCl}$ y hospitalaria queda reflejada en la tabla 4, sin diferencias entre ambos grupos. Se pasó un cuestionario a estos pacientes, una vez pasado el episodio agudo, sobre complicaciones asociadas al soporte no invasivo, y la mayoría destacó la presencia de ruido relacionado con el sistema de CPAP, pero de manera más acusada con el casco (tabla 5), que les impedía dormir. Como otras complicaciones, una paciente presentó una infección de orina por Pseudomonas aeruginosa. 
Tabla 2 Parámetros hemodinámicos, ventilatorios y analíticos de los pacientes a su ingreso en UCI

\begin{tabular}{|c|c|c|}
\hline Paramétros fisiológicos & Muestra total $(n=10)$ & Muestra de estudio $(n=5)$ \\
\hline $\mathrm{TAM}, \mathrm{mmHg}$ & $98[87-117]$ & $110[85-119]$ \\
\hline Frecuencia cardíaca, Ipm & $127[95-141]$ & $105[78-141]$ \\
\hline Frecuencia respiratoria, rpm & $35[33-38]$ & $38[29-41]$ \\
\hline $\mathrm{pH}, \mathrm{mmHg}$ & $7,42[7,27-7,48]$ & $7,47[7,42-7,51]$ \\
\hline $\mathrm{pCO}_{2}, \mathrm{mmHg}$ & $40[35-51]$ & $37[34-46]$ \\
\hline $\mathrm{EB}, \mathrm{mmHg}$ & $1[-2-2]$ & $2[0-11]$ \\
\hline $\mathrm{HCO}_{3}, \mathrm{mmHg}$ & $25[23-26]$ & $26[24-33]$ \\
\hline $\mathrm{pO}_{2} / \mathrm{FiO}_{2}$ & $87[65-113]$ & $63[58-87]$ \\
\hline \multicolumn{3}{|c|}{ Radiografía de tórax, número cuadrantes (\%) } \\
\hline $1 / 4$ & $3(30)$ & 0 \\
\hline $2 / 4$ & $2(20)$ & $1(20)$ \\
\hline $3 / 4$ & $2(20)$ & $2(40)$ \\
\hline $4 / 4$ & $3(30)$ & $2(40)$ \\
\hline LDH, UI/l & 934 [448-2.503] & 934 [772-2.503] \\
\hline GOT, UI/l & $48[28-61]$ & $53[48-626]$ \\
\hline GPT, UI/L & $63[14-76]$ & $72[45-406]$ \\
\hline Leucocitos/ $\mu$ l & $13.150[4.375-18.125]$ & $4.400[4.150-9.750]$ \\
\hline Plaquetas/ $\mu \mathrm{l}$ & $185.500[128.750-274.500]$ & $165.000[131.000-238.000]$ \\
\hline Urea, mg/dl & $29[16-52]$ & $26[18-67]$ \\
\hline Creatinina, mg/dl & $0,9[0,7-1,3]$ & $1[0,7-1,2]$ \\
\hline PCR, mg/l & $93[35-260]$ & $95[54-360]$ \\
\hline PCT, ng/ml & $2,4[0,4-14,8]$ & $5,1[0,3-17,4]$ \\
\hline
\end{tabular}

Los datos expresan mediana [índice intercuartílico 25-75] si no mencionan otra cosa.

\section{Discusión}

Pese a la pequeña muestra analizada, podemos concluir que, según los resultados obtenidos, la aplicación de la VMNI en sus distintas modalidades, pero sobre todo la CPAP con dispositivo Helmet, ha sido efectiva en la mejoría de la IRA hipoxémica en este grupo de pacientes, lo que ha permitido evitar la intubación orotraqueal y las complicaciones que de ella devienen.
La situación de disfunción de un solo órgano (donde predominaba la IRA hipoxémica), nos hizo plantear como terapia inicial la VMNI, puesto que, en nuestro medio, es una pauta inicial de tratamiento en todos los pacientes en IRA, siempre que las condiciones clínicas lo permitan. Otro dato que declinó la balanza en favor del empleo de la VMNI fue la extraordinaria tolerancia clínica de estos enfermos a la hipoxemia que presentaban, lo que indicaba que existía una dicotomía entre la clínica y las pruebas gasométricas y

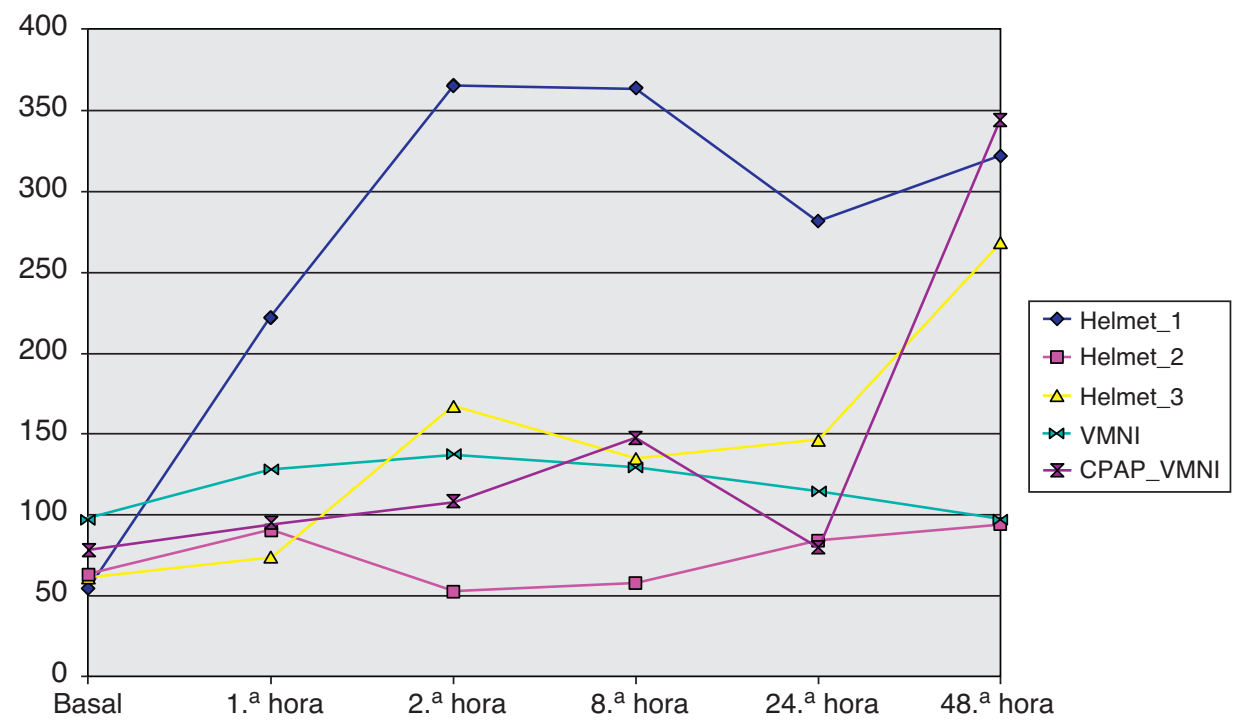

Figura 1 Evolución del cociente $\mathrm{pO}_{2} / \mathrm{FiO}_{2}$ durante el soporte ventilatorio. Se expresa en medianas. CPAP: presión continua vía aérea; VMNI: ventilación mecánica no invasiva. 


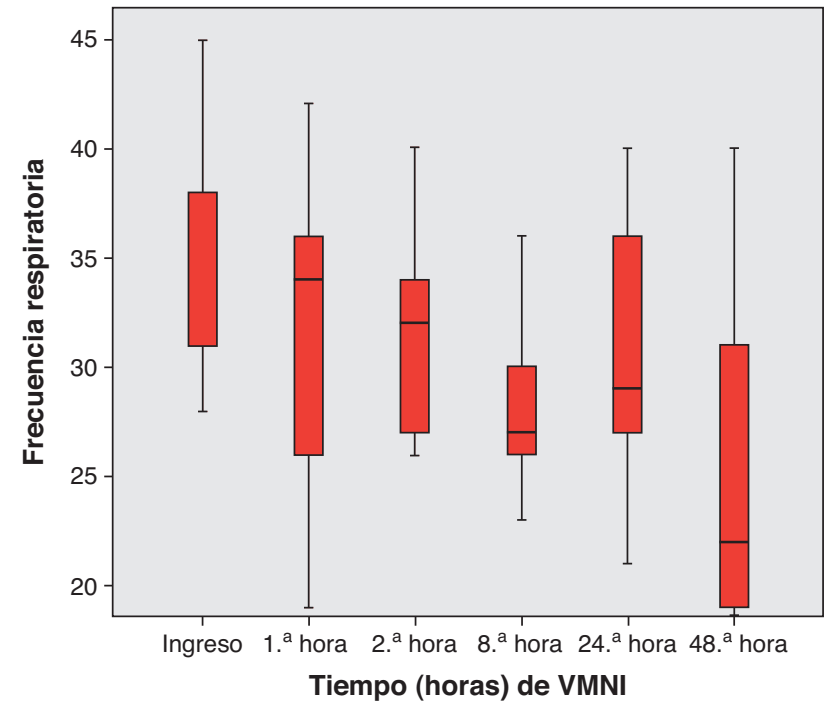

Figura 2 Evolución de la frecuencia respiratoria durante el soporte ventilatorio. Gráfica Box Plot con mediana e intervalo intercuartílico.

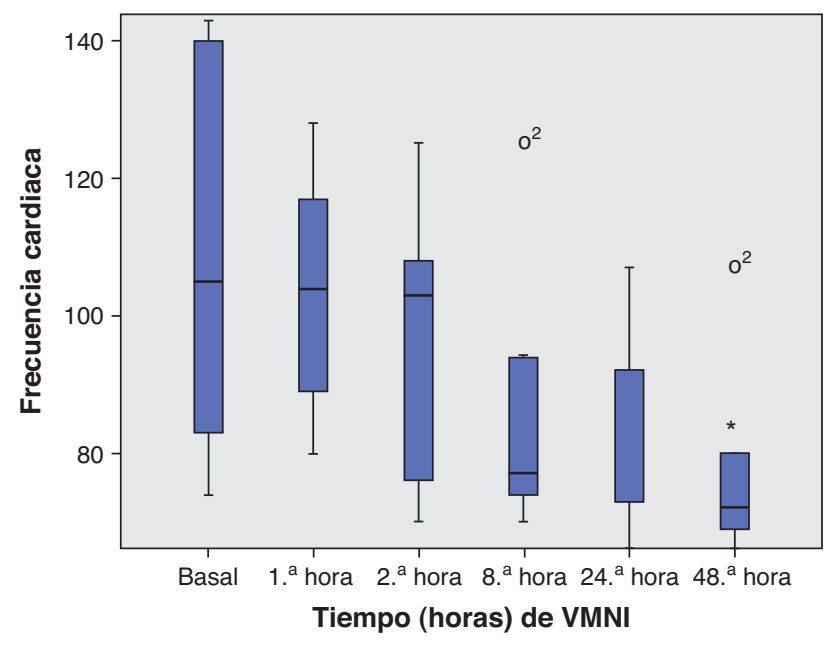

Figura 3 Evolución de la frecuencia cardíaca durante el proceso ventilatorio. * $\mathrm{p}<0,05$ por la prueba de Friedman. Gráfica Box Plot con mediana e intervalo intercuartílico. (o) Outliers, casos con valor entre 1,5 y 3 la longitud de la caja desde su borde superior o inferior.

Tabla 3 Retraso de inicio de síntomas hasta ingreso en hospital y UCI

\begin{tabular}{lll}
\hline & $\begin{array}{l}\text { Muestra } \\
\text { total }(\mathrm{n}=10)\end{array}$ & $\begin{array}{l}\text { Muestra } \\
\text { de estudio } \\
(\mathrm{n}=5)\end{array}$ \\
\hline $\begin{array}{l}\text { Tiempo inicio síntomas-ingreso } \\
\text { hospital, días }\end{array}$ & $3[2-6]$ & $5[2-7]$ \\
$\begin{array}{l}\text { Tiempo inicio síntomas-ingreso } \\
\text { en UCl, días }\end{array}$ & $4[2-8]$ & $5[2-9]$ \\
\hline Los datos expresan mediana [índice intercuartílico 25-75].
\end{tabular}

Tabla 4 Duración de soporte ventilatorio, estancia en UCI y hospitalaria

\begin{tabular}{lcc}
\hline & $\begin{array}{l}\text { Muestra } \\
\text { total }(\mathrm{n}=9)\end{array}$ & $\begin{array}{l}\text { Muestra de } \\
\text { estudio }(\mathrm{n}=5)\end{array}$ \\
\hline Duración ventilación, días & $6[3-11]$ & $6[4-11]$ \\
Estancia UCl, días & $8[5-16]$ & $9[7-11]$ \\
Estancia hospitalaria, días & $18[14-27]$ & $17[14-23]$ \\
\hline
\end{tabular}

Los datos expresan mediana [índice intercuartílico 25-75].

radiológicas. Frente a la situación de hipoxemia grave producida por infiltrados difusos en varios campos pulmonares, se producía un fenómeno de tolerancia clínica por parte de estos pacientes, muy superior a enfermos con el mismo grado de hipoxemia causada por neumonía o SDRA, dado que enfermos aquejados por este tipo de procesos suelen cursar con importante trabajo respiratorio.

Finalmente, fue la falta de signos evidentes de fatiga muscular lo que nos inclinó a emplear sistemas de presión continua en la vía aérea (CPAP) en lugar de dispositivos no invasivos con dos niveles de presión (excepto en 2 casos). A través de la producción de una presión supraatmosférica a lo largo de todo el ciclo respiratorio, los sistemas de CPAP aumentan la capacidad residual funcional y desplazan la sustancia que inunda el alveolo hacia sus partes más periféricas, aumentando la superficie de intercambio gaseoso, lo que se traduce en reducción de la hipoxemia. Aunque la CPAP no es un sistema de VMNI propiamente dicho, se suele englobar dentro ella debido a que cumple la mayoría de las propiedades de la VMNI.

El uso de VMNI como primera opción terapéutica se contrapone con los estudios publicados ${ }^{1,2,10-12}$ donde el porcentaje de pacientes ventilados con VMNI fue relativamente pequeño (entre el 6 y el 33\%), y con una tasa de fracaso superior al $85 \%^{2}$. Esta disparidad de resultados quizá podría deberse al mayor número de fallo de órganos que presentan estos pacientes en el momento de iniciar la VMNI, en comparación con la situación de casi exclusivo fallo respiratorio de nuestros pacientes en el momento de instaurar el soporte no invasivo (medido por SOFA), junto con el hecho de que fueron remitidos directamente desde urgencias y con un inicio inmediato de la VMNI.

Pese a haberse publicado artículos que muestran resultados satisfactorios con el empleo de la VMNI en pacientes hipoxémicos ${ }^{4-6}$, incluso con SDRA, los malos resultados obtenidos de la experiencia de la VMNI, tanto en Canadá ${ }^{2}$ como en Estados Unidos, han condicionado una actitud escéptica hacia el uso de la VMNI, y este hecho queda reflejado en las recomendaciones de soporte ventilatorio ${ }^{13}$. El otro factor que influiría en el rechazo de la VMNI es el riesgo añadido de diseminación del virus a través de las microgotas y contagio entre el personal sanitario ${ }^{13}$, tal y como ocurrió en

Tabla 5 Complicaciones asociadas a la VMNI

\begin{tabular}{lc}
\hline Ruido asociado a VMNI, n (\%) & $4(80)$ \\
Conjuntivitis, n (\%) & $2(40)$ \\
Distensión gástrica, n (\%) & 0 \\
Claustrofobia, n (\%) & $2(40)$ \\
\hline
\end{tabular}


la epidemia de Canadá de 2003, donde el 72\% del personal sanitario se vio infectado. En nuestra experiencia, no fue notificado ningún contagio en el personal por las extremas medidas de seguridad tomadas en el manejo de estos enfermos.

En la actualidad disponemos de varias interfaces o mascarillas para realizar la VMNI: mascarillas faciales, orofaciales o cascos (helmet). Los cascos son sistemas cerrados, a modo de escafandra de buzo, hechos de polivinilo transparente, que comparten los beneficios de la VMNI y, además, son mucho más confortables porque no producen lesiones sobre el puente de la nariz ${ }^{14}$. Diversas publicaciones han demostrado un éxito del dispositivo Helmet en pacientes con IRA hipoxémica ${ }^{14-16}$, tanto en modalidad de VMNI como con uso de sistemas de flujo continuo (CPAP). Teniendo en consideración que en pacientes hipoxémicos se preveía una ventilación prolongada, sumado a que no mostraban signos evidentes de trabajo respiratorio ni hipercapnia, optamos por utilizar dichos dispositivos que, a la postre, resultaron efectivos. El principal inconveniente del sistema Helmet empleando CPAP es la retención de anhídrido carbónico ${ }^{17}$, pero en ninguno de nuestros pacientes se produjo tal evento, ya que se emplearon flujos altos de aire-oxígeno ${ }^{17}$. Se pudo apreciar una baja tasa de complicaciones, y fue fundamentalmente el ruido generado por el sistema de flujo continuo del sistema CPAP el principal inconveniente para estos pacientes.

La pregunta que uno se formula es la siguiente: ¿la VMNI es la panacea?, la respuesta es, taxativamente, no, ya que está demostrado por estudios de cohortes que, en los pacientes con SDRA ventilados con VMNI en unidades especializadas, el porcentaje de fracaso se sitúa en un $50 \%{ }^{6}$. La extraordinaria evolución de nuestros pacientes nos hace reflexionar; si nuestros pacientes hubiesen sido intubados directamente ante la presencia de un patrón radiológico de SDRA junto con un cociente $\mathrm{pO}_{2} / \mathrm{FiO}_{2}$ inferior a 200 , ¿el resultado habría sido el mismo?, quizá no. Y si se hubiese empleado más la VMNI, ¿los resultados hubiesen sido los publicados?, es una cuestión a debatir.

¿A qué enfermos nos plantearíamos un soporte no invasivo de entrada? Quizá a los pacientes conscientes y colaboradores, sin situación de shock y fallo casi exclusivo respiratorio, que no presenten signos evidentes de trabajo respiratorio (que puedan requerir una intubación orotraqueal inmediata) ni una situación de acidosis metabólica láctica que indicaría una hipoperfusión tisular. En cuanto al tiempo que debemos esperar para plantear la intubación en un paciente que no experimenta un clara mejoría gasométrica, es un tema no aclarado, más aún cuando está demostrado que un retraso en la intubación aumenta la mortalidad ${ }^{18}$. Antonelli et $\mathrm{al}^{6}$ establecieron como factores de predicción de fracaso de la VMNI la presencia de mayor gravedad de los pacientes (SAPS II >34) y ausencia de mejora del cociente $\mathrm{pO}_{2} / \mathrm{FiO}_{2}>175$ a los $60 \mathrm{~min}$ de iniciar la VMNI. Quizá $1 \mathrm{~h}$ para retirar la VMNI sea muy escasa para poder valorar su efecto en estos pacientes, por ello se puede ampliar a varias horas (no hay un tiempo establecido), pero siempre evaluando los demás parametros ventilatorios, hemodinámicos y metabólicos.

Nuestros resultados apoyan el uso de VMNI (y sobre todo de CPAP con sistema Helmet) en pacientes con IRA hipoxémica por el nuevo virus de la gripe $A(\mathrm{H} 1 \mathrm{~N} 1)$, por lo que en una previsión futura de nuevas pandemias podría difundirse el empleo de estos dispositivos para poder enfrentarse a ellas como medida terapéutica eficaz en casos seleccionados.

\section{Conflicto de intereses}

Los autores declaran no tener conflictos de intereses.

\section{Bibliografía}

1. Kumar A, Zarychanski R, Pinto R, Cook D, Marshall J, Lacroix J, et al. Critically ill Patients with 2009 Influenza A (H1N1) infection in Canada. JAMA. 2009;302:1872-9.

2. Domínguez-Cherit G, Lapinsky SE, Macias A, Pinto R, Espinosa-Perez L, De la Torre A, et al. Critically ill patients with 2009 Influenza A (H1N1) infection in Mexico. JAMA. 2009;302:1880-7.

3. Rello J, Rodríguez A, Ibañez P, Socias L, Cebrian J, Marques A, et al. Intensive Care Adults patients with severe respiratory failure cuased by Influenza A (H1N1)v in Spain. Crit Care. 2009;13:R148.

4. Antonelli M, Conti G, Rocco M, Bufi M, De Blasi RA, Vivino G, et al. A comparison of noninvasive positive-pressure ventilation and conventional mechanical ventilation in patients with acute respiratory failure. N Engl J Med. 1998;339:429-35.

5. Ferrer M, Esquinas A, León M, González G, Alarcón A, Torres A. Noninvasive ventilation in severe hypoxemic respiratory failure. Am J Respir Crit Care Med. 2003;168:1438-44.

6. Antonelli M, Conti G, Esquinas A, Montini L, Maggiore SM, Bello $G$, et al. A multiple-center survey on the use in clinical practice of noninvasive ventilation as a first-line intervention for acute respiratory distress syndrome. Crit Care Med. 2007;35:18-25.

7. International Consensus Conference in Intensive Care Medicine. Non-invasive positive pressure ventilation in acute respiratory failure. Intensive Care Med. 2001;27:166-78.

8. Nava S, Hill N. Non-Invasive ventilation in acute respiratory failure. Lancet. 2009;374:250-9.

9. Mandell LA, Wunderink RG, Anzueto A, Bartlett JG, Campbell GD, Dean NC, et al. Infectious Diseases society of America/American Thoracic Society consensus guidelines on the management of community-acquired pneumonia in adults. Clin Infect Dis. 2007;44 Suppl 2:S27-72.

10. Rodríguez A, Socías L, Guerrero JE, Figueira JC, González N, Maraví-Poma E, et al. Gripe A pandémica en una unidad de cuidados intensivos: experiencia en España y Latinoamerica (Grupo Español de Trabajo de Gripe A Grave/Sociedad Española de Medicina Intensiva, Crítica y Unidades Coronarias). Med Intensiva. 2010;34:87-94.

11. Paredes $G$, Cevallos $C$. Síndrome de distress respiratorio agudo, durante la pandemia de Influenza A H1N1 en Ecuador. Med Intensiva. 2010;34:310-7.

12. Estenssoro E, Ríos FG, Apezteguía C, Reina R, Neira J, Ceraso DH, et al. Pandemic 2009 Influenza A in Argentina. Am J Respir Crit Care Med. 2010;182:41-8.

13. Ramsey CD, Funk D, Miller RR, Kumar A. Ventilator management for hypoxemic respiratory failure attibutable to $\mathrm{H} 1 \mathrm{~N} 1$ novel swine origin influenza virus. Crit Care Med. 2010;38 Suppl 4:S58-65.

14. Antonelli M, Conti G, Pelosi P, Gregoretti C, Pennisi MA, Costa R, et al. New treatment of acute hypoxemic respiratory failure: Noninvasive pressure support ventilation delivered by helmet-A pilot controlled trial. Crit Care Med. 2002;30:602-8.

15. Tonnelier JM, Prat G, Nowak E, Goetghebeur D, Renault A, Boles JM, et al. Noninvasive continuous positive airway 
pressure ventilation using a new helmet interface: a case-control prospective pilot study. Intensive Care Med. 2003;29:2077-80.

16. Principi T, Pantanetti S, Catani F, Elisei D, Gabbanelli V, Pelaia A, et al. Non-invasive continuous positive airway pressure delivered by helmet in hematological malignacy patients with hypoxemic acute respiratory failure. Intensive Care Med. 2004;30:147-50.
17. Taccone P, Hess D, Caironi P, Bigatello LM. Continuous positive airway pressure delivered with a "helmet": effects on carbon dioxide rebreathing. Crit Care Med. 2004;32: 2090-6.

18. Esteban A, Frutos-Vivar F, Ferguson N, Arabí Y, Apezteguía C, González $M$, et al. Noninvasive Positive-Pressure Ventilation for Respiratory Failure after extubation. N Engl J Med. 2004;350:2452-60. 